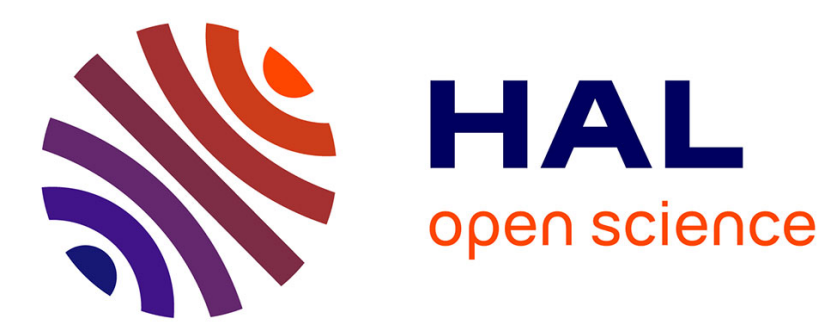

\title{
Sparse BSS from Poisson Measurements
}

\author{
Jerome Bobin, I El Hamzaoui, A Picquenot, F. Acero
}

\section{To cite this version:}

Jerome Bobin, I El Hamzaoui, A Picquenot, F. Acero. Sparse BSS from Poisson Measurements. 2019. hal-02083157

\section{HAL Id: hal-02083157 \\ https://hal.science/hal-02083157}

Preprint submitted on 28 Mar 2019

HAL is a multi-disciplinary open access archive for the deposit and dissemination of scientific research documents, whether they are published or not. The documents may come from teaching and research institutions in France or abroad, or from public or private research centers.
L'archive ouverte pluridisciplinaire HAL, est destinée au dépôt et à la diffusion de documents scientifiques de niveau recherche, publiés ou non, émanant des établissements d'enseignement et de recherche français ou étrangers, des laboratoires publics ou privés. 


\title{
Sparse BSS from Poisson Measurements
}

\author{
J.Bobin, I. El hamzaoui, A. Picquenot and F. Acero
}

\begin{abstract}
Blind source separation (BSS) aims at recovering signals from linear mixtures. This problem has been extensively studied when the noise is additive and Gaussian. This is however not the case when the measurements follow Poisson or shot noise statistics, such as in low photon count optics or in high-energy astronomical imaging. To that purpose, we introduce a novel BSS algorithm coined pGMCA (poisson-Generalized Morphological Component Analysis) that specifically tackles the blind separation of sparse sources from measurements following Poisson statistics. The proposed algorithm builds upon Nesterov's smoothing technique to define a smooth approximation of sparse BSS, with a data fidelity term derived from the Poisson likelihood. This allows to design a block coordinate descent-based minimization procedure with a simple choice of the regularization parameter. Numerical experiments have been carried out that illustrate the robustness of the proposed method with respect to Poisson noise. The pGMCA algorithm has been further evaluated in a realistic astrophysical $\mathrm{X}$-ray imaging setting.
\end{abstract}

\section{NOTATIONS AND DEFINITIONS}

In the following, matrices will be written in bold face. The $i$-th row of the matrix $\mathbf{X}$ will be denoted as $\mathbf{X}_{i}$ and its $t$ th sample $\mathbf{X}_{i}[t]$. This matrix will be composed as follows: $\mathbf{X}=\mathbf{X}_{i}[t]$. Each column of $\mathbf{X}$ is denoted by $\mathbf{X}[t]$

The Frobenius norm of the matrix $\mathbf{X}$ is defined as: $\|\mathbf{X}\|_{F}=$ $\sqrt{\operatorname{Trace}\left(\mathbf{X X}^{T}\right)}$. The element-wise or Hadamard product will be denoted by $\odot$ and the element-wise division by $\oslash$.

The oblique set $\mathcal{O}_{b}$ is defined as matrices $\mathbf{X}$ whose columns have unit $\ell_{2}$ norms: $\mathcal{O}_{b}=\left\{\mathbf{X} ; \forall t,\|\mathbf{X}[t]\|_{\ell_{2}}=1\right\}$. The nonnegative orthant $K^{+}$is the set composed of matrices with nonnegative entries: $K^{+}=\left\{\mathbf{X} ; \forall i, t, \mathbf{X}_{i}[t] \geq 0\right\}$. For the sake of simplicity, $\mathbf{X} \in K^{+}$will be equivalently written as $\mathbf{X} \geq 0$.

\section{INTRODUCTION}

Multichannel data are composed of $m$ observations $\mathbf{X}_{i}$, each of which is made of $t$ samples. According the standard instantaneous linear mixture, each observation is described as a linear combination of $n$ elementary sources or components $\mathbf{S}_{j}$. A classical assumption is that the data are corrupted with additive -generally Gaussian- noise, which leads to the following matrix formulation:

$$
\mathbf{X}=\mathbf{A S}+\mathbf{N}
$$

where $\mathbf{X} \in \mathbb{R}^{m \times t}$ is the observation matrix, $\mathbf{S} \in \mathbb{R}^{n \times t}$ the source matrix, $\mathbf{A} \in \mathbb{R}^{m \times n}$ the mixing matrix and $\mathbf{N} \in \mathbb{R}^{m \times t}$ for the noise contribution. In this context, BSS aims at recovering both the mixing matrix $\mathbf{A}$ and the sources $\mathbf{S}$ from the data $\mathbf{X}$ only. This is essentially an unsupervised matrix factorization; being ill-posed it requires additional assumptions

J. Bobin and I. El hamzaoui are with CEA, IRFU, DEDIP, 91191 Gif-surYvette Cedex, France. A. Picquenot and F. Acero are with CEA, IRFU, DAp, 91191 Gif-sur-Yvette Cedex, France about the sources and/or the mixing matrix, such as statistical independence [1], non-negativity [2] or sparsity [3], [4] to only name three.

However, the above linear mixture model does not describe precisely the kind of data that are commonly found in low photon count imaging, such as in X-ray observations [5]. For that purpose, one needs to account for the exact statistics of the measurements, which precisely follow a Poisson distribution. Hence, the data $\mathbf{X}$ are only defined statistically from the "noiseless" mixtures AS; the probability for a given sample to take the value $\mathbf{X}_{i}[t]$ is then given by the Poisson law:

$$
\mathcal{P}\left(\mathbf{X}_{i}[t] \mid[\mathbf{A S}]_{i}[t]\right)=\frac{e^{-[\mathbf{A S}]_{i}[t]}[\mathbf{A} \mathbf{S}]_{i}[t]^{\mathbf{X}_{i}[t]}}{\mathbf{X}_{i}[t] !}
$$

where $[\mathbf{A S}]_{i}[t]$ is the samples of the matrix AS located at the $i$-th row and $t$-th column.

In this case, the observations do not rigorously follow the linear mixture model since the Poisson stochastic process does not preserve linearity; it only holds on average. For a single stochastic realisation, fitting for the linear mixture model without accounting for the exact measurement stochastic distribution is very likely to yield imperfect separation.

\section{In the ICA and NMF frameworks}

To tackle BSS from Poisson measurements, a straightforward approach consists in maximizing the likelihood of the mixture variables. In the case of Poisson statistics, this amounts to minimizing the Kullback-Leibler divergence between the data $\mathbf{X}$ and the mixture model $\mathbf{A S}$ with respect to $\mathbf{A}$ and $\mathbf{S}$. This has been investigated both in the scope of Independent Component Analysis (ICA - [6]) and Nonnegative Matrix Factorization (NMF - [2], [7]), where it generally refers to robust BSS. In this setting, the mixing matrix and the sources are estimated by minimizing the $\beta$ divergence $D_{\beta}$ between the data $\mathbf{X}$ and the model $\mathbf{A S}$ under the assumption that both $\mathbf{A}$ and $\mathbf{S}$ are non-negative:

$$
\min _{\mathbf{A} \geq 0, \mathbf{S} \geq 0} D_{\beta}(\mathbf{X}, \mathbf{A S}) \text {. }
$$

Multiplicative algorithms are customarily used as they implicitly encode the non-negativity of the mixture parameters. For $\beta=1$, the $\beta$-divergence is exactly the Kullback-Leibler divergence. In this case, the above problem is similar to a maximum likelihood estimate of $\mathbf{A}$ and $\mathbf{S}$ assuming that the data are distributed according to a Poisson distribution, which is the case that will be considered in the rest of this article. Sparse NMF has also been investigated with Euclidean [8], [9] as well as $\beta$-divergence [10] data fidelity terms. The main limitation of these algorithms is that sparsity is enforced in the sample domain, which is not suitable for a large variety 
of applications, where sparsity needs to be enforced in a transformed domain such as in astrophysical imaging.

\section{In the sparse BSS framework}

In the scope of sparse BSS, the sources are assumed to admit a sparse distribution in some signal representation $\boldsymbol{\Phi}$. More precisely, each source $\left\{s_{j}\right\}_{j=1, \cdots, n}$ can be described in some basis, waveform dictionary or signal representation $\boldsymbol{\Phi}$ as $s_{j}=\alpha_{j} \boldsymbol{\Phi}$. Then, sparse BSS algorithms seek the mixing matrix and the sources so that the latter are mutually the sparsest. It has long been highlighted that sparse modeling allows to compressively encode the information content of the sources to be estimated. Improving the contrast between these sources eventually leads to enhanced separation processes [11]-[14] as well as increased robustness with respect to noise [14]. However, in the case of Poisson, the noise variance is proportional to the square root of the Poisson mean, i.e. the product AS. Since sparse BSS is mainly sensitive to the most salient features of the sources [14], [15], the Poisson nature of the noise will largely impact the performances of standard sparse BSS methods.

Contributions While sparse BSS has been successful in various applications (see [16] for an example in astrophysics), to the best of our knowledge, the blind separation of sparse sources has not been investigated when the data follow a Poisson distribution. The proposed approach first consists in performing the separation by putting together sparse regularization and a data fidelity term derived from the Poisson likelihood. This raises two important challenges: i) most modern-day algorithms for sparse matrix factorization require the data fidelity term to be smooth [17], which is not the case for the Poisson likelihood about 0 , ii) in the low statistics regime (i.e. when the Poisson mean takes small values), the Poisson likelihood, taken as a function of either the sources $\mathbf{S}$ or the mixing matrix $\mathbf{A}$, becomes highly ill-conditioned, which largely hinders the speed of convergence of most algorithms, and eventually hampers the separation quality. In this article, these challenges are addressed by making use of a BCD algorithm (Block-coordinate descent - [18]) along with a smooth approximation of the Poisson likelihood based on Nesterov's smoothing technique [19]. The proposed method coined Poisson Generalized Morphological Component Analysis (pGMCA) is detailed in Section II. Numerical experiments are carried out on simulated and realistic X-ray astrophysical data in Section III.

\section{SPARSE BSS FROM POISSON MEASUREMENTS}

\section{A. From additive Gaussian noise to Poisson statistics}

In the next, we assume that each source admits a sparse representation in some signal representation $\boldsymbol{\Phi}$, which is described precisely with the synthesis sparse signal model: $\forall j=1, \cdots, n, s_{j}=\alpha_{j} \Phi$. In the GMCA algorithm, the sparsity of the expansion coefficients $\alpha_{j}$ is enforced by minimizing its re-weighted $\ell_{1}$ norm with $p \leq 1$ :

$$
\min _{\mathbf{A} \in \mathrm{Ob}, \mathbf{S}}\|\boldsymbol{\Lambda} \odot \alpha\|_{\ell_{p}}+\frac{1}{2}\|\mathbf{X}-\mathbf{A} \alpha \boldsymbol{\Phi}\|_{F}^{2},
$$

In practice, the choice $p=1$ is adopted. The matrix $\Lambda$ contains the regularization parameters as well as potentially weights in the spirit of re-weighted $\ell_{1}$-norm regularization [20]. The last term is the data fidelity term, which would be identical to the anti-log likelihood of the variables $\mathbf{A}$ and $\mathbf{S}$ for additive Gaussian noise. The oblique constraint alleviates the standard scale indeterminacy between the mixing matrix and the sources.

Switching from additive Gaussian to Poisson noise naturally requires substituting the quadratic Frobenius norm with the Poisson anti-log likelihood:

$$
\min _{\mathbf{A} \in \mathcal{C}, \mathbf{S} \geq 0}\left\|\boldsymbol{\Lambda} \odot \mathbf{S} \boldsymbol{\Phi}^{T}\right\|_{\ell_{1}}+\mathcal{L}(\mathbf{X} \mid \mathbf{A}, \mathbf{S}) .
$$

The second term is the neg-loglikelihood of the variables $\mathbf{A}$ and $\mathbf{S}$ :

$$
\begin{aligned}
\mathcal{L}(\mathbf{X} \mid \mathbf{A}, \mathbf{S}) & =\sum_{i, t}[\mathbf{A} \mathbf{S}]_{i}[t]-\mathbf{X}_{i}[t] \log \left([\mathbf{A} \mathbf{S}]_{i}[t]\right) \\
& =\mathbf{A} \mathbf{S}-\mathbf{X} \odot \log (\mathbf{A} \mathbf{S})
\end{aligned}
$$

The mixing matrix is further imposed to have non-negative entries. This entails that the mixing matrix belongs to $\mathcal{C}$, which is defined as the intersection between the oblique set and the non-negative orthant: $\mathcal{C}=\mathrm{Ob} \cap K^{+}$. As well, the sources are constrained to have non-negative entries.

Finding a solution to the problem in Eq. 2 raises several challenges:

- A multi-convex problem: the problem is not convex but convex with respect to each variable $\mathbf{A}$ and $\mathbf{S}$ assuming the other one is fixed. This class of optimization problems are dubbed multi-convex problems. In [14], [15], the GMCA algorithm has been built as a projected ALS algorithm (Alternate Least-Squares), which has been showed to provide a reliable (i.e. robust with respect to initialization) and effective proxy to approximate solution of the problem in 2. This choice was in part motivated by its low computational cost. However, when the non-negativity of the sources and the mixing matrices needs to be enforced, it has been emphasized in [21] that an ALS-based approach like GMCA is not appropriate.

Several alternatives have been recently proposed to tackle multi-convex problems such as the Block-CoordinateDescent (BCD - [17], [18]) or Proximal Alternating Linear Minimization (PALM - [17], [22]), sequentially optimizes over each variable independently.

- Non-differentiability and unbounded Hessian of the data fidelity term: in Equation 2, the term $\mathcal{L}(\mathbf{X} \mid \mathbf{A}, \mathbf{S})$ is not smooth about 0 , which rigorously excludes the use of PALM, which requires a smooth data fidelity term. For non-zero Poisson mean (i.e. non-zero mixed samples), the Poisson neg-loglikelihood is differentiable but its Hessian scales like $1 \oslash(\mathbf{A S} \odot \mathbf{A S})$; it is therefore unbounded at the vicinity of 0 . Furthermore, since the Hessian can take large values, the step size required for 
any minimiser based on gradient descent would take dramatically small values in the low statistics regime. This would lead to dramatically slow minimisation schemes.

\section{B. A smooth approximation of data fidelity term}

So as to alleviate the aforementioned obstacles, we propose to make use of a smooth approximation of the data fidelity term $\mathcal{L}$. For that purpose, we proposing implementing the smoothing technique introduced by Nesterov in [19]. Following Nesterov's approach, a smooth approximate $\mathcal{L}_{\mu}$ of the convex function $\mathcal{L}$ can be built from its Fenchel dual $\mathcal{L}^{\star}[23]$ as follows:

$$
\mathcal{L}_{\mu}(\mathbf{X} \mid \mathbf{Y})=\inf _{\mathbf{U}}\langle\mathbf{Y}, \mathbf{U}\rangle-\mathcal{L}^{\star}(\mathbf{X} \mid \mathbf{U})-\mu g(\mathbf{U}),
$$

where $\langle\mathbf{Y}, \mathbf{U}\rangle$ stands for the scalar product between the matrices $\mathbf{Y}$ and $\mathbf{U}$. The smoothing parameter $\mu$ is a strictly positive real-valued scalar. The function $g$ is strongly convex; in practice it is simply chosen as $g(\mathbf{U})=\|\mathbf{U}\|_{F}^{2}$.

It can be shown that the dual function of the Poisson negloglikelihood takes the following form:

$$
\mathcal{L}^{\star}(\mathbf{X} \mid \mathbf{U})=\mathbf{X} \odot(1-\log (\mathbf{X}))-\mathbf{X} \odot(1-\log (\mathbf{U}))
$$

Following the definition in Equation $5, \mathcal{L}_{\mu}$ is differentiable for any non-negative entry and admits a Lipschitz gradient $\nabla \mathcal{L}_{\mu}$. Some simple algebra yields the following expression:

$$
\begin{aligned}
\nabla \mathcal{L}_{\mu}(\mathbf{X} \mid \mathbf{Y})= & \operatorname{Argmax}_{\mathbf{U}}\langle\mathbf{Y}, \mathbf{U}\rangle-\mathcal{L}^{\star}(\mathbf{U})-\mu\|\mathbf{U}\|_{F}^{2} \quad(6) \\
= & \frac{1}{2 \mu}(\mathbf{Y}+\mu) \odot \cdots \\
& \left.\cdots\left[1-\sqrt{1-4 \mu(\mathbf{Y}-\mathbf{X}) \oslash(\mathbf{Y}+\mu)^{2}}\right] 8\right)
\end{aligned}
$$

The square root and power of two are also taken entry-wise. The choice of the smoothing parameter $\mu$ will be discussed in Section II-D.

\section{C. pGMCA: a BCD-based algorithm}

In the next, we investigate the use of the Block-CoordinateDescent algorithm (see [18] - BCD) to tackle the following approximate problem:

$$
\min _{\mathbf{A} \in \mathcal{C}, \alpha \mathbf{\Phi} \geq 0}\|\boldsymbol{\Lambda} \odot \alpha\|_{\ell_{1}}+\mathcal{L}_{\mu}(\mathbf{X} \mid \mathbf{A}, \alpha \boldsymbol{\Phi}),
$$

where $\mathbf{S}=\alpha \boldsymbol{\Phi}$. Based on BCD, the pGMCA algorithm sequentially updates each variable $\mathbf{S}$ and $\mathbf{A}$ so that each iteration $(k)$ can be described with the following two steps.

1) Description of the update of $\mathrm{S}$ : For a fixed current estimate $\hat{\mathbf{A}}^{(k-1)}$ of $\mathbf{A}$, the sources are updated by solving the problem:

$$
\min _{\alpha}\|\boldsymbol{\Lambda} \odot \alpha\|_{\ell_{1}}+\iota_{K^{+}}(\alpha \mathbf{\Phi})+\mathcal{L}_{\mu}(\mathbf{X} \mid \mathbf{A}, \alpha \mathbf{\Phi}),
$$

which can be more generally described as follows:

$$
\min _{\mathbf{Y}} \sum_{r=1}^{2} \mathcal{F}_{r}(\mathbf{Y})+\mathcal{D}(\mathbf{Y}),
$$

where both $\mathcal{F}_{1}$ and $\mathcal{G}_{2}$ are non-smooth regularization terms and $\mathcal{D}$ is smooth with Lipschitz gradient. This convex problem is composed of a smooth data fidelity term and two nonsmooth regularization functional, which makes the framework of proximal algorithms [24] perfectly adapted to build a minimizer.

In Eq. 10, the function $\mathcal{F}+\mathcal{G}$ has a well-defined proximal operator but it does not admit a closed-form expression. This precludes the use of standard proximal algorithms such as the Forward-Backward Splitting algorithm (FBS - [25]). In this context, the Generalized Forward-Backward Splitting algorithm (G-FBS - [26]) is way more adapted since it allows to call independently the proximal operators of individual regularization terms. A generic pseudo-code of the G-FBS algorithm is given in 1 . The implementation for the above problem is detailed in the next paragraphs.

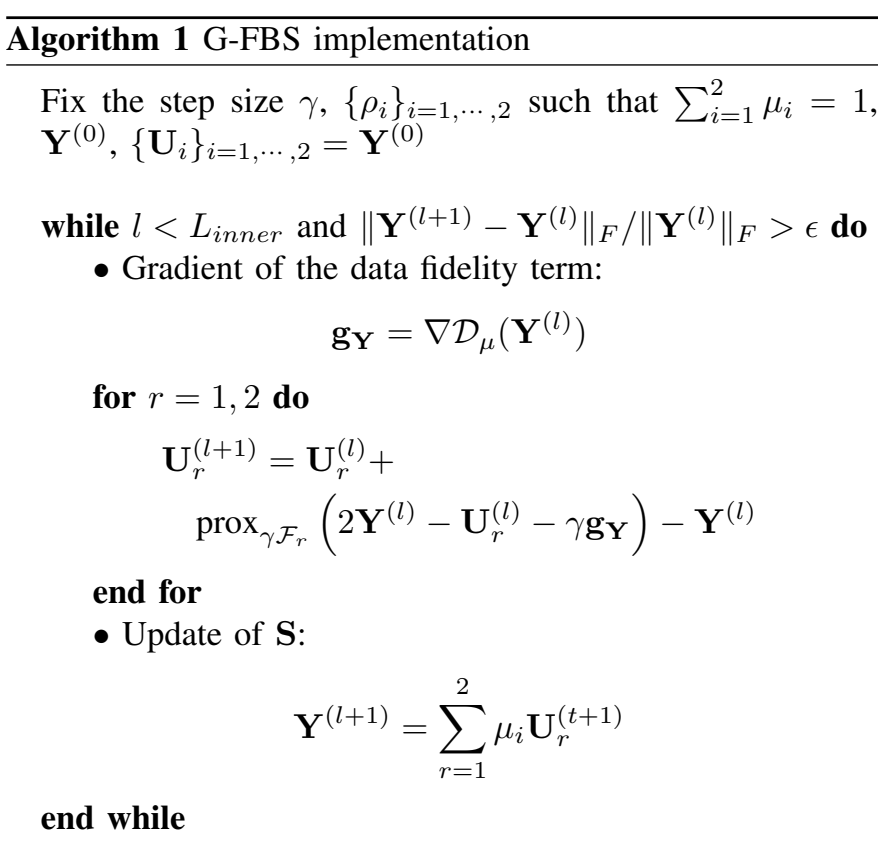

Details of the G-FBS implementation are given below:

- Gradient expression: the gradient of the data fidelity term with respect to the sources is given by: $\mathbf{g}_{\mathbf{S}}=\mathbf{A}^{T} \nabla \mathcal{L}_{\mu}(\mathbf{X}, \mathbf{A S})$. It is Lipschitz with constant $L_{\mathbf{S}}=\left\|\mathbf{A} \mathbf{A}^{T}\right\|_{2} / \mu$.

- Sparse regularization: The proximal operator of the sparse regularization $\|\boldsymbol{\Lambda} \odot(.)\|_{\ell_{1}}$ is well-known to be the softthresholding operator that applies independently on each entry of the input argument $\mathbf{Y}[i, j]$ :

$$
\begin{aligned}
& \operatorname{prox}_{\boldsymbol{\Lambda} \odot(.) \|_{\ell_{1}}}(\mathbf{Y}[i, j])= \\
& \left\{\begin{array}{cc}
\mathbf{Y}[i, j]-\boldsymbol{\Lambda}[i, j] \operatorname{sign}(\mathbf{Y}[i, j]) & \text { if }|\mathbf{Y}[i, j]|>\boldsymbol{\Lambda}[i, j] \\
0 & \text { otherwise }
\end{array}\right.
\end{aligned}
$$

- Positivity constraint: Depending on the properties of the signal representation $\Phi$, the proximal operator of the sparse regularization $i_{(.) \Phi \geq 0}($.$) can take different$ expressions.

When $\boldsymbol{\Phi}$ is orthogonal (e.g. Discrete Cosine Transform, 
Biorthogonal Wavelets, etc.), it is straightforwardly defined as:

$$
\operatorname{prox}_{i_{(.) \Phi \geq 0}}(\mathbf{Y})=[\mathbf{Y} \boldsymbol{\Phi}]_{+} \boldsymbol{\Phi}^{T}
$$

If $\boldsymbol{\Phi}$ is a tight frame, for which $\boldsymbol{\Phi}^{T} \boldsymbol{\Phi}=\mathbf{I}$ (e.g. Undecimated wavelets [27], Curvelets [28], etc.), it can be shown to take the following closed-form formulation [21]:

$$
\operatorname{prox}_{i_{(\cdot) \Phi \geq 0}}(\mathbf{Y})=\mathbf{Y}+[-\mathbf{Y} \boldsymbol{\Phi}]_{+} \boldsymbol{\Phi}^{T}
$$

The hyperparameters of the G-FBS include weights $\left\{\rho_{i}\right\}_{i=1,2}$ that impose a relative weight between the two intermediate variables $\mathbf{U}_{i=1,2}$, and therefore the related penalisations. In the next, these weights have both been fixed to $1 / 2$; other choices did not lead to significant differences of the speed of convergence. The stepsize $\gamma$ is fixed to $\gamma=\frac{\mu}{\left\|\mathbf{A}^{T} \mathbf{A}\right\|_{2}}$.

2) Description of the update of $\mathbf{A}$ : For a fixed current estimate $\hat{\mathbf{S}}^{(k)}$ of $\mathbf{S}$, the mixing matrix is updated by solving the following problem:

$$
\min _{\mathbf{A} \in \mathcal{C}} \mathcal{L}_{\mu}(\mathbf{X} \mid \mathbf{A}, \mathbf{S}) .
$$

Since the set $\mathcal{C}$ is the intersection of the oblique set $\mathcal{O}_{b}$ and the non-negative orthant, the above problem can be rewritten by introducing the characteristic function $\iota$ of these sets:

$$
\min _{\mathbf{A}} \iota_{(.) \in \mathcal{C}}(\mathbf{A})+\mathcal{L}_{\mu}(\mathbf{X} \mid \mathbf{A}, \mathbf{S}) .
$$

This problem can be solved using the Forward-Backward splitting algorithm [25], or more precisely one of its accelerated variant FISTA [29]. Details of the implementation of the FISTA implementation are given below:

- Gradient expression: the gradient of the data fidelity term with respect to the sources is given by: $\mathbf{g}_{\mathbf{A}}=\nabla \mathcal{L}_{\mu}(\mathbf{X}, \mathbf{A S}) \mathbf{S}^{T}$. It is Lipschitz with constant $L_{\mathbf{A}}=\left\|\mathbf{S}^{T} \mathbf{S}\right\|_{2} / \mu$.

- Constraints: The mixing matrix is constrained to belong to the set $\mathcal{C}$, which is the intersection of the oblique set and the non-negative orthant.

Strictly speaking, the oblique set is not convex and does not rigorously admit a proximal operator. However, if none of the columns of some matrix $\mathbf{Y}$ vanishes, its orthogonal projection onto $\mathcal{O}_{b}$ is uniquely defined. The proximal operator of its characteristic function $\iota_{(.)} \in \mathcal{O}_{b}$ will be defined as: $\operatorname{prox}_{(.) \in \mathcal{O}_{b}}(\mathbf{Y})=\left[\mathbf{Y}^{k} /\left\|\mathbf{Y}^{k}\right\|_{\ell_{2}}\right]$. The proximal operator of the positivity constraint boils down to the orthogonal projection onto the positive orthant can be shown to take the following closed-form formulation [21]: $\operatorname{prox}_{(.) \in K^{+}}(\mathbf{Y})=[\mathbf{Y}]_{+}$.

From the above definitions, it can be showed that the proximal operator of its characteristic function $\iota_{(.) \in \mathcal{C}}$ can be defined as the composition of $\operatorname{prox}_{(.) \in K^{+}}$and $\operatorname{prox}_{(.) \in \mathcal{O}_{b}}$ :

$$
\operatorname{prox}_{(.) \in \mathcal{C}}=\operatorname{prox}_{(.) \in \mathcal{O}_{b}} \circ \operatorname{prox}_{(.) \in K^{+}}
$$

The main hyperparamter of the FBS algorithm is the step-size $\gamma$, which is fixed to $\gamma=\frac{\mu}{\left\|\mathbf{S S}^{T}\right\|_{2}}$. The iterations stop whenever the relative variation between consecutive iterations of $\mathbf{A}$ is below $\epsilon=10^{-6}$.

\section{Implementation details}

a) Initialization: : The problem in Eq. 9 being multiconvex, the $\mathrm{BCD}$ algorithm turns to be highly sensitive to the initial point. In the meantime the GMCA algorithm has long been advocated as an algorithm that is robust with respect to the initialization [30]. Therefore, the pGMCA algorithm will use the output of GMCA as first guess estimate for the mixing matrix and the sources.

b) Smoothing parameter: : The smoothing parameter $\mu$ has a direct impact on the quality of approximation of $\mathcal{L}$ and its gradient $\nabla \mathcal{L}$. Obviously, when $\mu$ tends to $0, \mathcal{L}_{\mu}$ converges towards $\mathcal{L}$. More specifically, Equation 6 reveals that $\mu$ mainly applies as an offset on $\mathbf{Y}$ in the expression of the gradient of $\mathcal{L}_{\mu}$. Therefore $\mu$ has to be directly compared to the value of the Poisson average (or average number of counts). In the next paragraph, we discuss how it can be chosen along with the regularization parameters.

c) Regularization parameters: : In practice, the $\mathrm{BCD}$ turns out to be quite sensitive to the choice of the regularization parameters. In the current setting, the role played by the matrix $\Lambda$ is twofold: i) it implements a re-weighted scheme by imposing a relative weighting of the entries of $\mathbf{S}$ in the signal representation $\boldsymbol{\Phi}$ (see [20]), and ii) it has also to be set so as to correctly reject noise contributions. Consequently, the matrix $\Lambda$ is designed based on the first guess $\bar{\alpha}$ (e.g. the GMCA estimate of the sources in the signal representation) so that for each entry $(i, j)$ :

$$
\begin{aligned}
\forall i, j ; \boldsymbol{\Lambda}[i, j] & \left.=\lambda_{i} w_{[} i, j\right] \\
& =\lambda_{i} \frac{\nu}{\nu+\frac{|\bar{\alpha}[i, j]|}{\max _{j}|\bar{\alpha}[i, j]|}} .
\end{aligned}
$$

This formulation allows to split the regularization parameter into two terms: i) the re-weighting contribution $\left.w_{[} i, j\right]$ that takes its values in the range $[0,1]$ and $\nu=1 e-3$, and ii) the noise-related part $\lambda_{i}$ that is only source-dependent. Hence, reweighting term is defined as a modulation with respect to a standard noise-based thresholding.

Let us recall that the $\boldsymbol{\Lambda}$ eventually plays the role of a threshold in the signal representation. The denoising power of the sparse regularization therefore depends on its ability to throw away noise in the G-FBS update of $\mathbf{S}$. Since thresholding applies on a gradient descent update of the sources as displayed in 1, the thresholds $\left\{\lambda_{i}\right\}_{i=1, n}$ are chosen based on the gradient about the initial point:

$$
\lambda_{i}=\tau \cdot \operatorname{MAD}\left(\nabla_{\mathbf{S}} \mathcal{L}_{\mu}\left(\mathbf{A}^{(0)}, \mathbf{S}^{(0)}\right) \boldsymbol{\Phi}^{T}\right),
$$

where the Median-Absolute-Deviation (MAD) is an empirical estimate of the standard deviation of the noise measured on the gradient in the sparse domain $\boldsymbol{\Phi}$. Interestingly, while this approach is known to well-suited for additive Gaussian noise, such a strategy provides good empirical separation results in the Poisson case for $\tau=1$. Indeed, the distribution of the noise contribution in the sparse representation will highly depend on the approximation of the gradient of $\mathcal{L}_{\mu}$ with respect to $\mathbf{S}$. For small values of $\mu$ - typically smaller than the mean value of the data $\overline{\mathbf{X}}-$ Fig. 1 shows that the gradient will be highly asymmetric about 0 . It will tend to be 


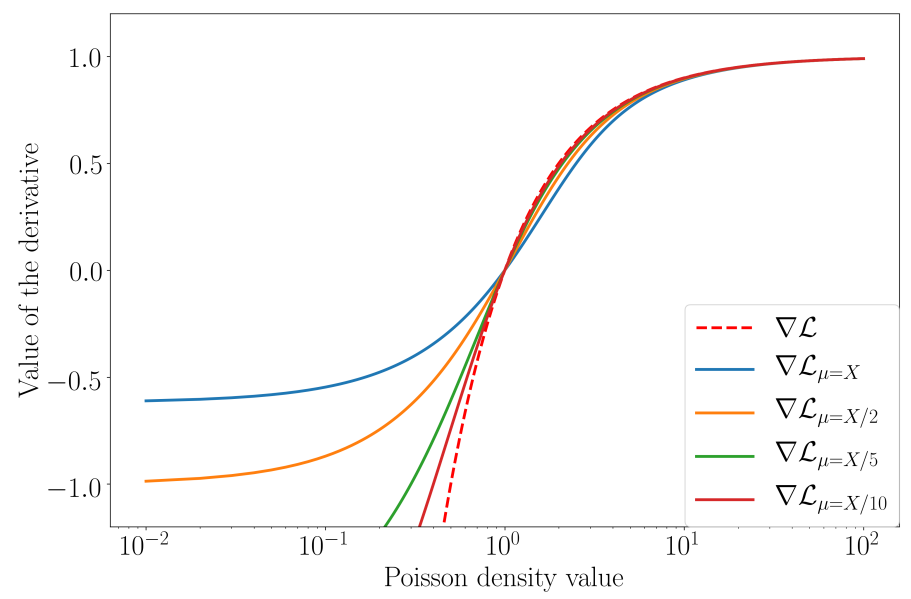

Figure 1: Approximations of $\nabla \mathcal{L}$ for different values of $\mu$.

more symmetric for $\mu \simeq \overline{\mathbf{X}}$. Furthermore, Fig.2 displays the histogram of the gradient term in the signal representation $A^{\star T} \nabla \mathcal{L}_{\mu} \boldsymbol{\Phi}^{T}$ (which is computed about the true mixing matrices and sources) for two values $\mu=\overline{\mathbf{X}}$ and $\mu=\overline{\mathbf{X}} / 10$ along with their Gaussian best fit. This figure emphasizes that a more symmetric smooth approximation tends to have a more Gaussian distribution in the transformed domain, which makes the proposed MAD-based strategy efficient in this regime. Consequently, $\mu=\overline{\mathbf{X}}$ is set for the next experiments.

d) Stopping criterion and number of iterations: : The number of outer iterations $L_{\text {outer }}$ is fixed 100; the number of inner iterations $L_{\text {inner }}$ (i.e. number of iterations to update $\mathbf{S}$ and $\mathbf{A}$ for each outer iteration) is fixed to 1000 . The overall algorithm stops when the Frobenius norm between two consecutive estimates of the mixing matrix $\left\|\mathbf{A}^{(k+1)}-\mathbf{A}^{(k)}\right\|_{F}$ is lower than $\epsilon=10^{-6}$.

\section{NUMERICAL EXPERIMENTS}

\section{A. Experimental set-up}

In the following experiments, our prime intention is to investigate the performance of the poisson GMCA algorithm with respect to standard matrix factorization algorithms. To that end, we first make use of synthetic data that mimic uni-dimensional spectroscopic data, which allows to perform Monte-Carlo simulations to assess the robustness of the different methods. The data are described as synthesized as follows:

- The sources $\left\{s_{j}\right\}_{j=1, \cdots, n}$ are $K$-sparse signals; each source $s_{j}$ has only $K$ non-zero entries out of $T$ entries. The activation of the entries of the sources will be distributed according to a Bernoulli process $\pi$ with parameter $\rho=0.02$ (i.e. the probability for a given sample to be non-zero is equal to $\rho$ ). Each source is made of $T=1024$ samples.

- the sources are convolved with a Laplacian kernel of full width at half height (FWHM) equal to 8 .

- The mixing matrix will be picked at random from a uniform distribution and processed so as to be nonnegative with a pre-defined condition number.
The simulated sources (resp. an example of mixture) are displayed in the right (resp. right) panel of Figure 3. Such sources admit a sparse representation in a frame of undecimated (translation invariant) 1D wavelets [31].

\section{B. Methods' description}

In addition to the proposed pGMCA algorithm, comparisons will be carried our with the following algorithms:

- The sparse NMF algorithm [10]. Leroux et al. proposed a fresh look at sparse NMF problems. Precisely, they introduce an improved multiplicative update algorithm that correctly impose both the non-negativity of the factors and the sparsity of the sources in the sample domain. The problem solved by the sNMF algorithm reads as:

$$
\min _{\mathbf{A} \geq 0, \mathbf{S} \geq 0} \lambda\|S\|_{\ell_{1}}+D_{\beta}(\mathbf{X}, \mathbf{A S}) .
$$

In the forthcoming numerical experiments, the simulated sources are mildly sparse in the sample domain; therefore it won't be a totally unreasonable assumption.

- The HALS algorithm [32]. The Hierarchical Alternate Least-Squares (HALS) algorithm is a NMF algorithm that minimizes a standard quadratic data fidelity term under positivity constraints. In constrast to other ALSbased methods, each source and its corresponding column of the mixing matrix are updated individually and sequentially, which leads to a simple and effective minimization scheme.

- The BetaNMF algorithm [2]. Originating from the hyperspectral imaging community, this method minimizes the Kullbback-Leibler divergence between the data and the model $\mathbf{A S}$ by further requiring the sources to be non-negative, and their samples lie in the simplex with almost pure pixels. The last assumption is however not valid in the following experiments. Instead, it is imposed that the columns of the estimated mixing matrix 

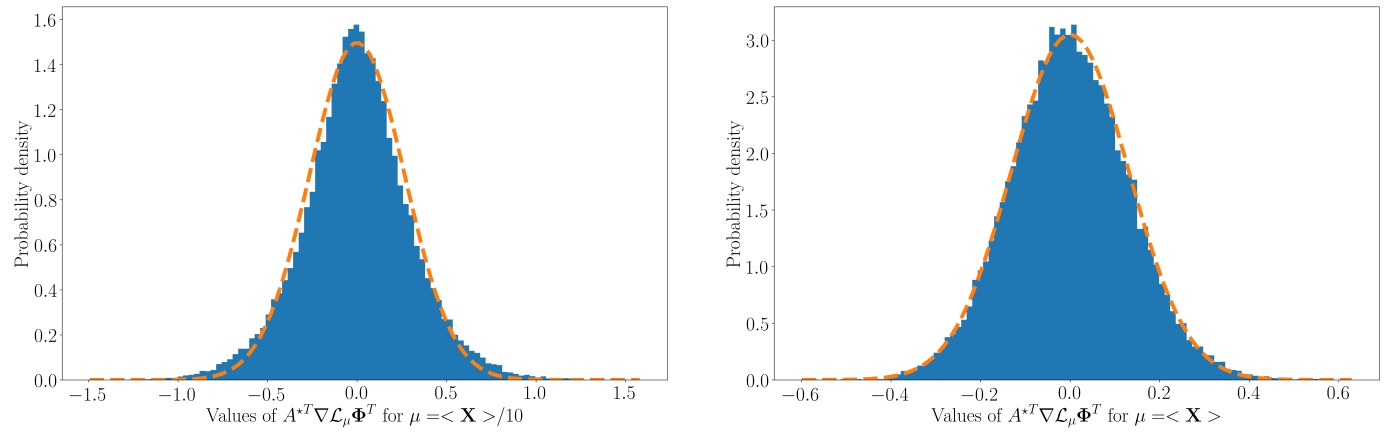

Figure 2: Histogram of the gradient of $\mathcal{L}_{\mu}$ with respect to $\mathbf{S}$ about the true input (mixing matrix and sources) and their Gaussian b best fit.

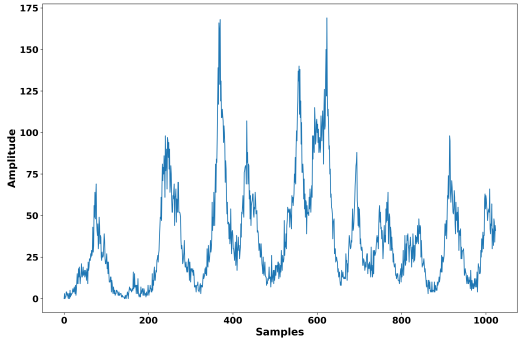

(a) Noisy measurement

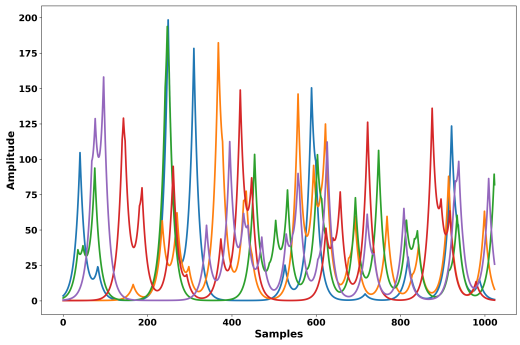

(b) Input sources

Figure 3: Examples of inputs. The average number of counts (Poisson average) is equal to 250.

have unit $\ell_{2}$ norm. The parameter $\beta$ of the $\beta$-divergence is set to 1 to minimize a Kullback-Leibler divergence, which is well suited for Poisson statistics.

- GMCA [4]. This a standard sparse BSS algorithm. Similarly to the pGMCA algorithm, the signal representation $\boldsymbol{\Phi}$ is chosen as the isotropic undecimated wavelet transform.

Unless stated differently, each single experimental result will be given as the mean over 25 Monte-Carlo simulations with different mixing matrix, sources and noise realisations.

\section{Comparison criteria}

A standard approach to evaluate the performances of matrix factorization algorithms is based on the decomposition of the estimated sources into different error terms [33]:

$$
s^{\text {est }}=s_{\text {target }}+s_{\text {interf }}+s_{\text {artefacts }},
$$

which can be interpreted as:

- $s_{\text {target }}$ is the projection of $s^{\text {est }}$ on the true source, which corresponds to the sought-after contribution

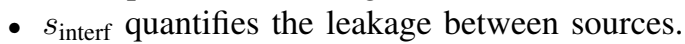

- $s_{\text {artefacts }}$ represents the remaining artifacts, which do not originate from interferences.

In contrast to the standard decomposition, the noise-related term has been left out since in the present case it is datadependent. Therefore, it cannot be identified as a distinct term. The Source to Distortion Ratio (SDR) is a combined quantity that gathers information from the different terms of the decomposition:

$$
\operatorname{SDR}\left(s^{\text {est }}\right)=10 \log _{10}\left(\frac{\left\|s_{\text {target }}\right\|_{2}^{2}}{\left\|s_{\text {interf }}+s_{\text {artefacts }}\right\|_{2}^{2}}\right) .
$$

Since these methods do no impose similar regularization on the sources, they are more fairly compared based on the quality of estimation of the mixing matrix. For that purpose, we make use of the mean and maximal spectral angular distance (SAD) between the estimated column $[\hat{\mathbf{A}}]^{j}$ and input column $\left[\mathbf{A}^{o}\right]^{j}$ of the mixing matrix:

$$
\mathrm{SAD}=\sum_{j=1}^{n} \cos ^{-1}\left(\left|\left\langle[\hat{\mathbf{A}}]^{j},\left[\mathbf{A}^{\star}\right]^{j}\right\rangle\right|\right) / n
$$

Results

Figure 4 displays the results for a single source given by the GMCA, pGMCA and HALS for a Poisson average of 250. The mixing matrix has been generated at random with a condition number of 2 . In this illustration, both the GMCA and pGMCA algorithms provide reasonable results. It can however be highlighted that the pGMCA algorithm yields a slightly less noisy estimate with better reconstructed peaks. This is particularly visible on peaks around 800th sample.

Evolution with respect to the mean number of counts $\delta$ : In this experiment, the performances of the BSS methods are evaluated with respect to the Poisson average (i.e. mean 


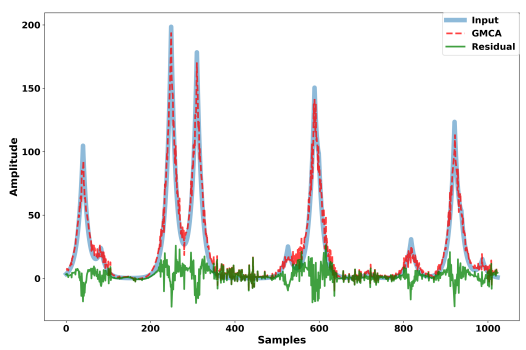

(a) GMCA

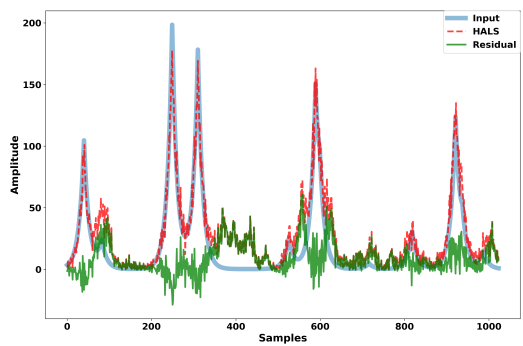

(b) HALS

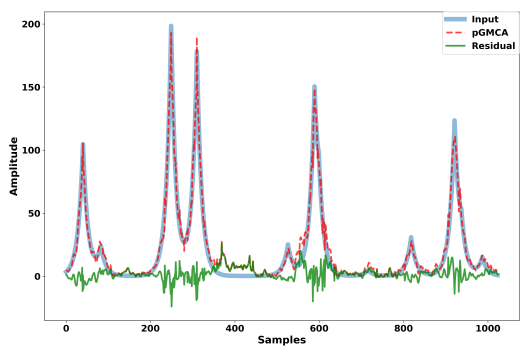

(c) pGMCA

Figure 4: Illustration of separation results. Solid blue: true source, dashed red: estimated source and solid green: residual error

number of counts) in range that goes from 5 to 5000 . The condition number of the generated mixing matrix is set to 2 . The left panel of Fig. 5 features the evolution of the mean SAD. The HALS and $\beta$-NMF provide results that do not vary much across the range which we considered. In contrast, sparse regularization (sNMF, GMCA and pGMCA) clearly helps improving the separation quality for an average $\delta$ larger than 50. As testified by the results provided by GMCA and pGMCA, the ability to model sparsity in the wavelet domain provide an extra decrease of the SAD that can reach more than one order of magnitude for $\delta \geq 500$. The pGMCA yields significantly better results for $\delta>100$, which highlights that the ability of the proposed algorithm to account for the Poisson nature of the noise. For low values of $\delta$, a significant number of data samples vanish, which explains the poor separation quality of most methods that lead to a mean SAD of about $10^{\circ}$.

Similar conclusions can be drawn by measuring the SDR of the estimated sources as shown in the right panel of Fig. 5. For $\delta<100$, all the methods yield a solution with a mean SDR that is below $15 \mathrm{~dB}$. For larger Poisson average, the pGMCA algorithm provides an improvement of about $2 \mathrm{~dB}$ with respect to the GMCA algorithm.

Evolution with respect to the condition number: In contrast to the standard additive Gaussian noise, the condition number of the mixing matrix plays an even more important role. Indeed, in the case of Poisson noise, the noise statistics is data dependent and its variance is equal to the noiseless mixture AS. When the condition number of the mixing matrix increases, the noise variance will therefore be dominated by the data contribution spanned by the singular vectors of $\mathbf{A}$ that correspond to the most dominant singular values. This will considerably limit the ability to discriminate between the sources.

To evaluate this effect, the left panel of Fig.6 shows the SAD as a function of the condition number of the mixing matrix A in the range $[1.2,9]$. It first shows that HALS and sNMF provide decent results only when the mixing matrix is close to orthogonality (i.e. condition number lower than 2). Both GMCA and pGMCA seem to be more robust with respect to conditioning of the mixing matrix with a SAD below $1^{\circ}$ when the condition number is lower than 5 . All the methods yield poor results when the mixing matrix condition number is larger than 8 . The right panel displays the evolution of the SDR of the estimated sources. This Fig. first confirms that when the condition number is larger than 2, the pGMCA algorithm leads to a gain of about $10 \mathrm{~dB}$ with respect to NMF-based methods and $2 \mathrm{~dB}$ with respect to the GMCA algorithm.

\section{Application in Astrophysics}

Description of the data: In this section, numerical experiments are carried out on simulations of astrophysical data that have been generated from real Chandra ${ }^{1}$ observations of the Cassiopea A supernova remnants. These data are composed of a linear combination of 3 astrophysical components: synchrotron emission, and 2 redshifted iron (Fe) emission lines. Mathematically, these emissions lines correspond to kronecker elements that are convolved with the telescope impulse response, which is Gaussian-shaped. These lines are centered about different energy values, which depend on the relative speed of propagation of each iron component as dictated by the Doppler effect. The synchrotron component has a power emission law. These components are

${ }^{1}$ http://chandra.harvard.edu/ 


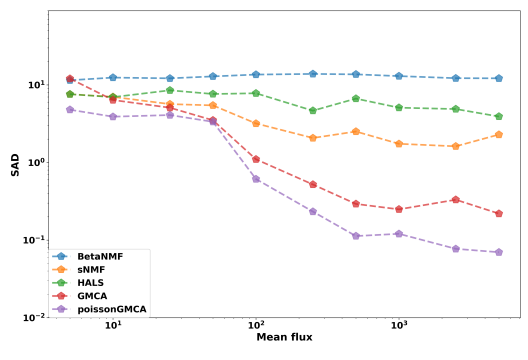

(a) Mean SAD

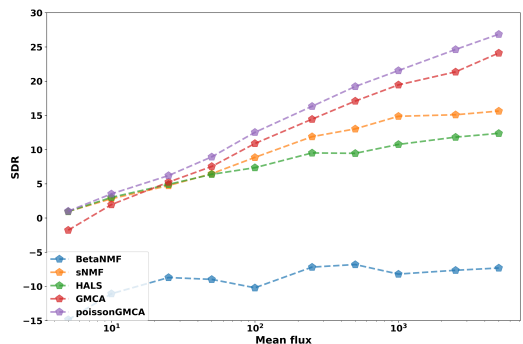

(b) Mean SDR

Figure 5: Evolution of the SAD (left panel) and SDR (right panel) as a function of the average of the Poisson distribution.

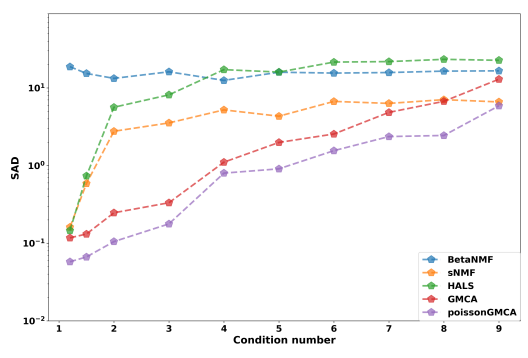

(a) Mean SAD

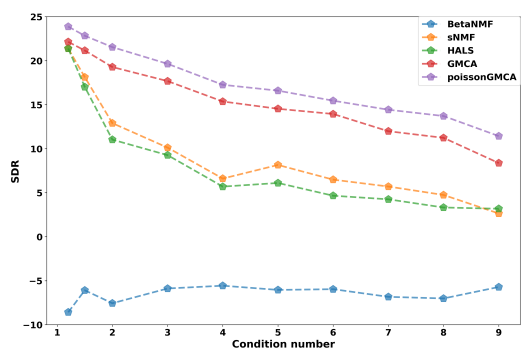

(b) Mean SDR

Figure 6: Evolution of the SAD (left panel) and SDR (right panel) as a function of the mixing matrix condition number.

representative of typical supernovae remnants in the energy band $5000-6000 \mathrm{eV}$ (electron-volt). In the next experiments, the data are composed of either 50 or 12 observations of size $128 \times 128$ pixels. The sources and their spectra are displayed in Fig. 7. Examples of mixtures are features in Fig.8.

\section{A. Illustration}

In the next experiments, the separation performances of the various methods will be evaluated based on the mean number of counts (i.e. average of the Poisson distribution) of the observed data $\mathbf{X}$, the number of observations $m$, and the relative intensity of the iron components with respect to the synchrotron emission. The data are generated so that the average of the Poisson measurements can be described with the following formula:

$$
\mathbf{X}^{\star}[i, t]=\frac{\rho\left(\mathbf{A}_{\mathrm{Fe}}^{\star} \mathbf{S}_{\mathrm{Fe}}^{\star}+\phi \mathbf{A}_{\text {sync }}^{\star} \mathbf{S}_{\text {Sync }}^{\star}\right)}{m t^{2} \sum_{i, t}\left[\left[\mathbf{A}_{\mathrm{Fe}}^{\star} \mathbf{S}_{\mathrm{Fe}}^{\star}+\phi \mathbf{A}_{\text {Sync }}^{\star} \mathbf{S}_{\text {sync }}^{\star}\right][i, t]\right.},
$$

where the scalar $\rho$ quantifies the mean number of counts per pixel in the observed data, and $\phi$ fixes the relative intensity between the synchrotron emission and the two Fe components. Studying the impact of these two parameters allows to consider various scenarios:

- Impact of the total intensity: making the total intensity $\rho$ vary allows to investigate the performances of the BSS methods in the low number of counts regime, where standard methods are less likely to provide good results, as well as larger intensity regimes, where the standard additive Gaussian noise assumption can be a good approximation.

- Impact of the relative intensity: in the current astrophysical setting, the synchrotron emission is a rather diffuse component that mainly plays the role of a background component with respect to the $\mathrm{Fe}$ components. Letting their relative intensities to change allows to investigate different observational regimes. More specifically, when the synchrotron emission dominates, the noise statistics are prominently related to this component due to the Poisson nature of the observations, which makes the recovery of the Fe components much more challenging.

In this paragraph, comparisons between the three components are performed with $\phi=5$ (i.e. the total intensity of the synchrotron emission is five times larger than the one of the other two components) and $\rho=5$ (i.e. the Poisson average per pixel is 5). The three panels of Fig.7 feature the input and estimated spectra of the three components (i.e. the columns of the mixing matrix). The top panels of 9 (resp. 9 and 11) show the sources estimated with the GMCA (resp. pGMCA and HALS) algorithm. The bottom panels of these figures feature the residuals with respect to the true sources. To make visual inspection easier, the color scales are similar for each method. These results first show that these three methods visually provide reasonable estimated sources. The GMCA algorithm yield a significant higher residual for the three sources. This is particularly true for the synchrotron emission that exhibits a leakage from the first iron source. Similarly, the 


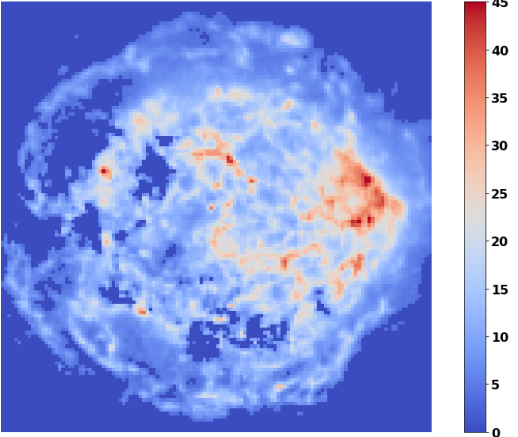

(a) Synchrotron

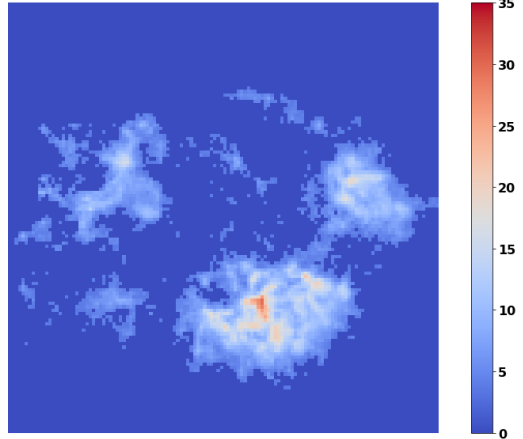

(b) $\mathrm{Fe}-1$

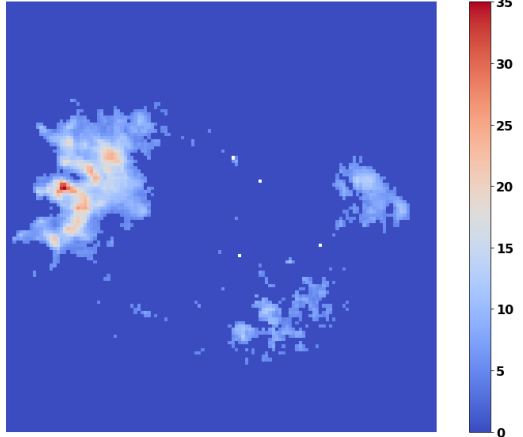

(c) $\mathrm{Fe}-2$

Figure 7: Input sources: synchrotron emission and the 2 iron emissions from top to bottom.

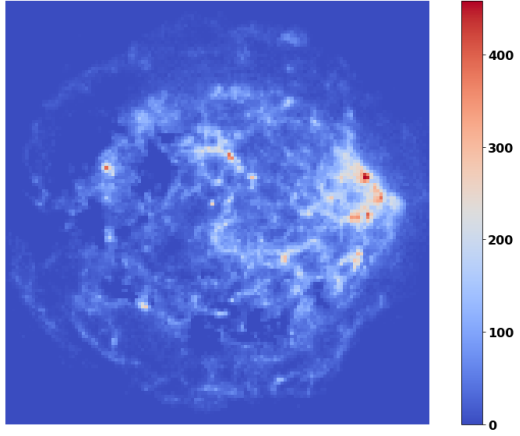

(a) $5279 \mathrm{eV}$

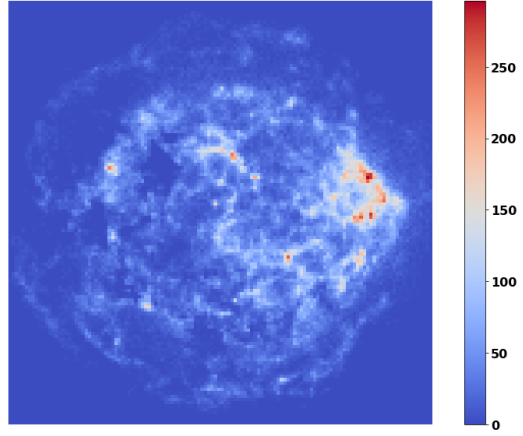

(b) $5548 \mathrm{eV}$

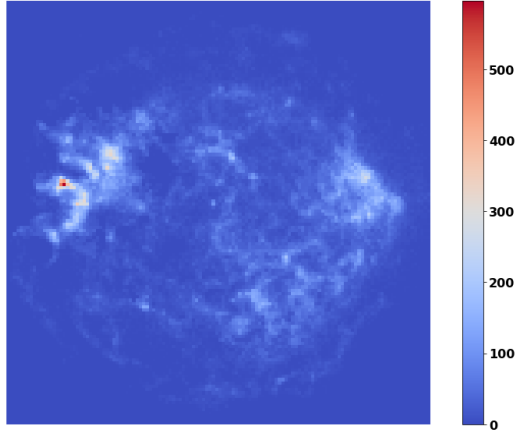

(c) $5817 \mathrm{eV}$

Figure 8: Examples of mixtures at 5279, 5548 and $5817 \mathrm{eV}$.

HALS algorithm shows a significant amount of synchrotron contamination in both the two redshifted iron sources. In contrast, the pGMCA algorithms leads to the lowest residues that visually correlates with the synchrotron emission. Since the synchrotron emission is dominant in this experiment, it is expected to be dominant as well in the noise. The noisy synchrotron imprint is therefore likely to be remainings of noise in the sources estimated with the pGMCA algorithm.

Figure 12 displays the estimated spectra for this experiment. This first confirms that the spectra of the two iron sources computed with the GMCA algorithm are not perfectly estimated; this is particularly the case for the first iron source, whose spectrum show a significant leakage of synchrotron at low energy. While the iron spectra estimated with the HALS seem to be very well estimated, it has to be highlighted that the synchrotron spectrum exhibits a significant bias in the range $[5600,6000] \mathrm{eV}$, where these sources peak. Keeping in mind that the synchrotron emission is dominant, this yields a much larger bias on the estimated sources as shown in Fig. 11. Finally, the pGMCA algorithm leads to the more robust estimates of the three spectra.

\section{B. Comparisons with respect to the mean flux}

In this section, we investigate the performances of BSS when the mean flux evolves. In these experiments the relative intensity parameter is fixed to $\phi=2$ and the number of observations is fixed to 12 . Figure 13 displays the evolution of the mean and maximum SAD when the mean flux varies from 0.5 to 100 .

In this experiment, the $\beta$-NMF and sNMF algorithms do not perform correctly. Similarly to the 1D case, the $\beta$-NMF does not seem to perform correctly. The sources are not sparse in the sample domain, which is a clear difference with the 1D case. However, as shown by the good separation quality of the HALS algorithm, non-negativity helps disentangling between the various sources. To that respect, the differences between sNMF and HALS is probably explained by the very good robustness of the HALS algorithm with respect to the initialization.

It can be pointed out that modeling the sparsity of the sources in the wavelet domain largely help improving the separation as shown by the results of GMCA and pGMCA in Fig. 13. Further accounting for the Poisson statistics of the observations yields SAD values lower than $0.1^{\circ}$ for mean number of counts larger than 8 . Interestingly, the results of the GMCA algorithm deteriorate significantly for values larger than 10. Since the measurements follow Poisson statistics, the signal-to-noise ratio (SNR) for a given pixel $t$ and observation $i$ is equal to $\sqrt{[\mathbf{A S}]_{i}[t]}$. As a consequence, large amplitude data samples have a smaller SNR. Since sparse BSS methods are more sensitive to the samples with the largest amplitudes 


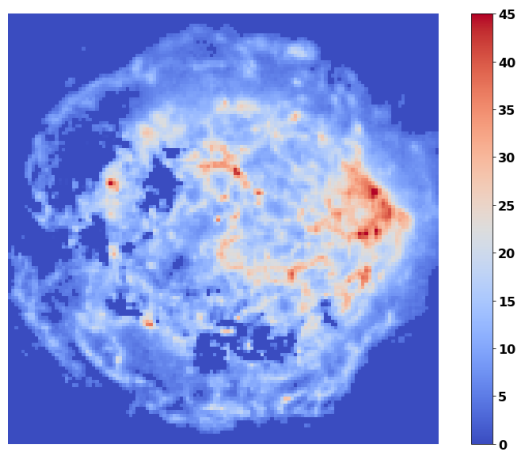

(a) Synchrotron

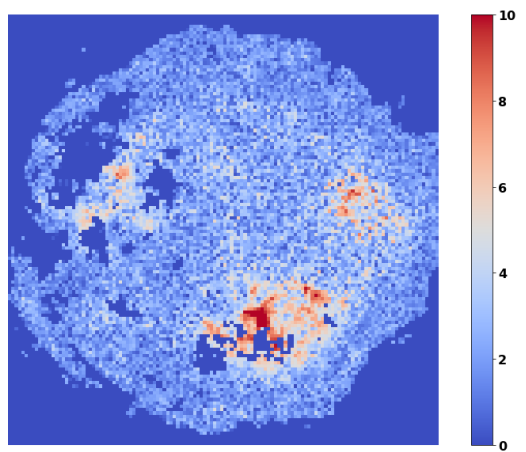

(d) Residual error

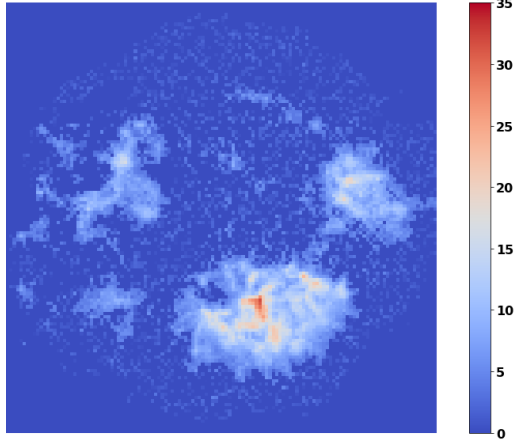

(b) Fe-1

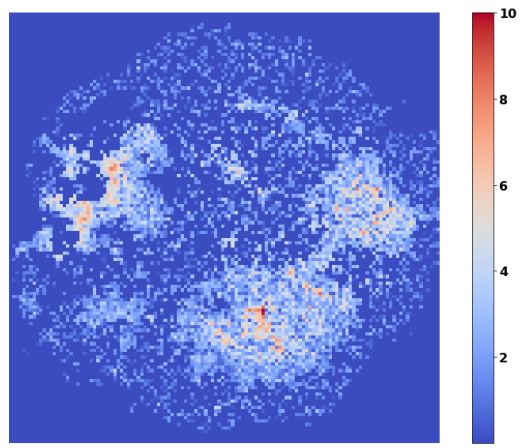

(e) Residual error

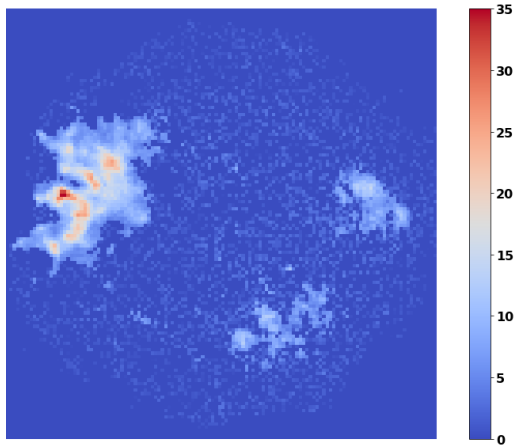

(c) Fe-2

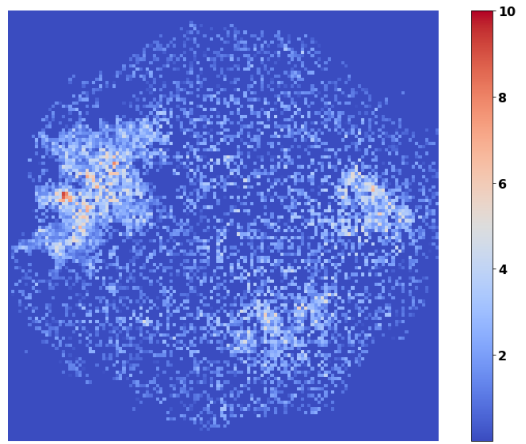

(f) Residual error

Figure 9: Sources estimated with GMCA.

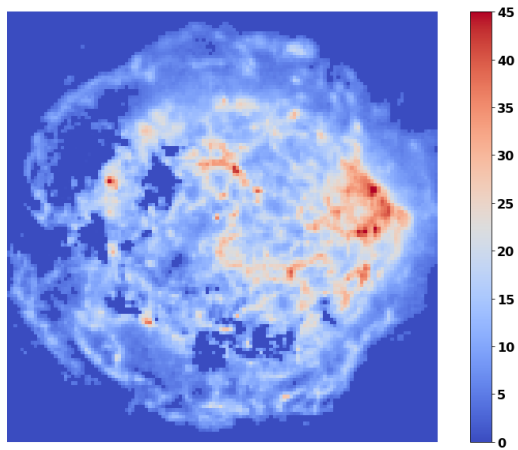

(a) Synchrotron

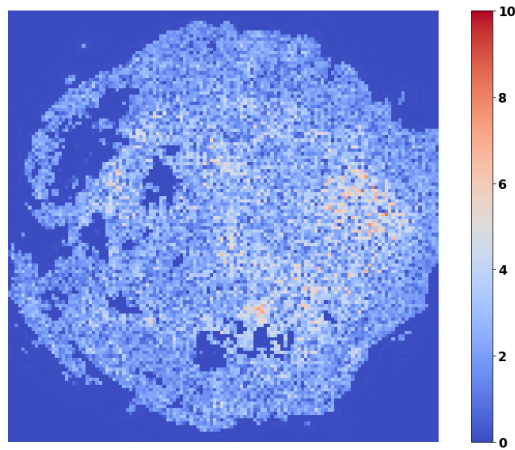

(d) Residual error

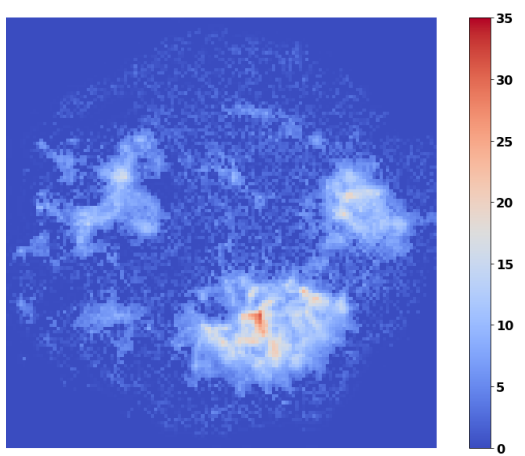

(b) $\mathrm{Fe}-1$

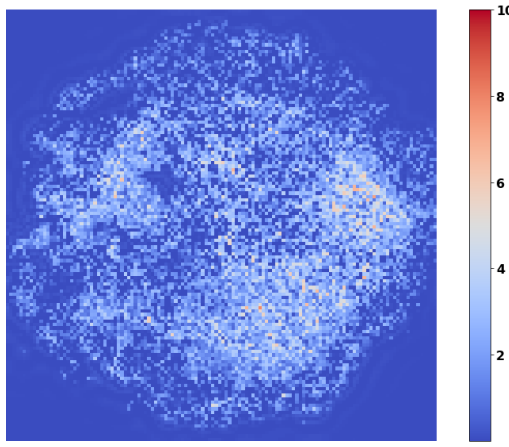

(e) Residual error

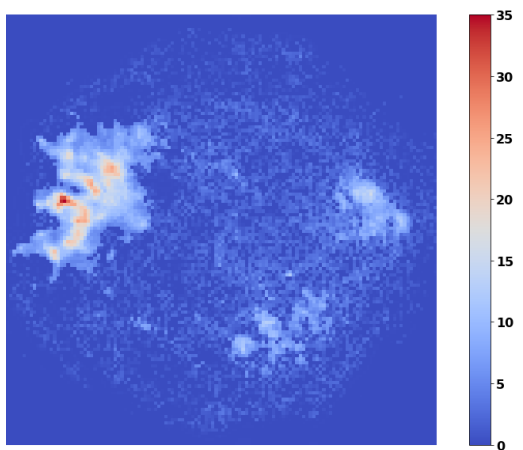

(c) $\mathrm{Fe}-2$

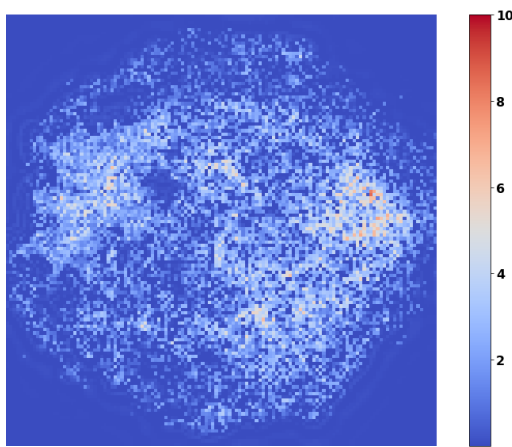

(f) Residual error

Figure 10: Sources estimated with pGMCA. 


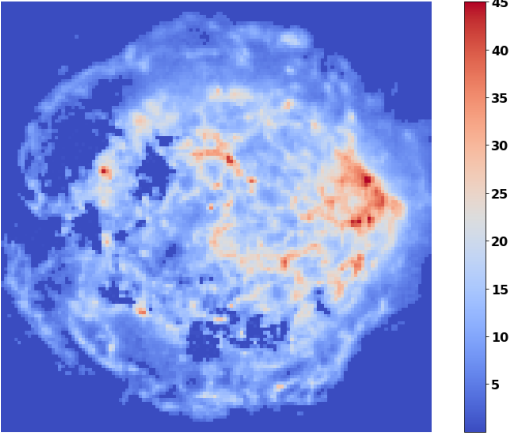

(a) Synchrotron

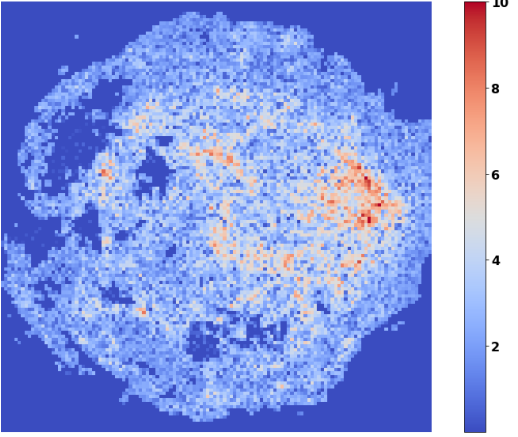

(d) Residual error

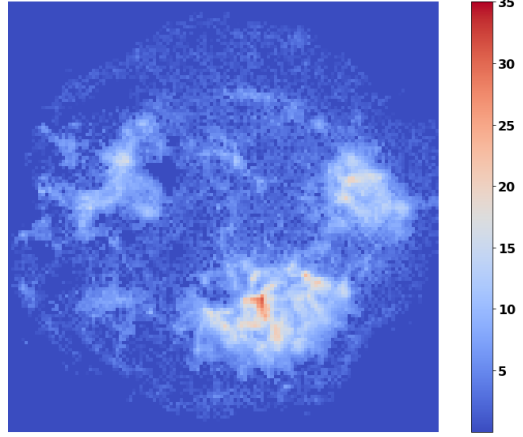

(b) $\mathrm{Fe}-1$

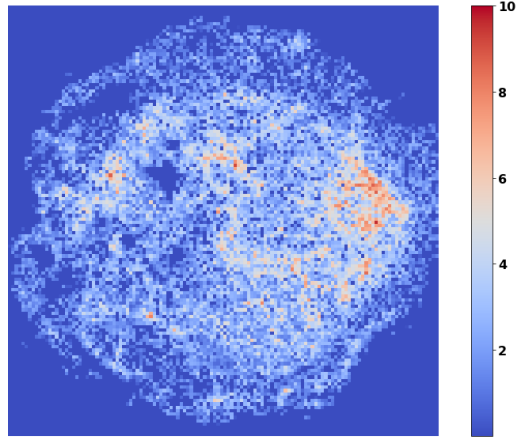

(e) Residual error

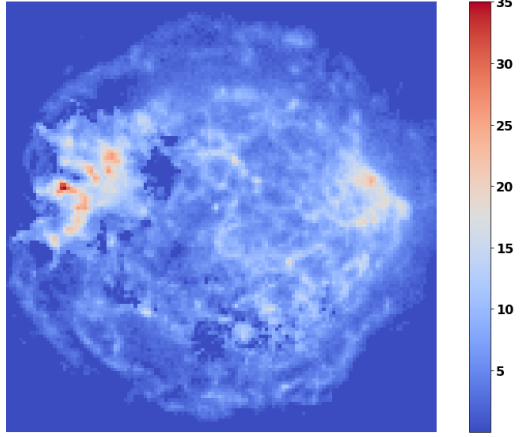

(c) $\mathrm{Fe}-2$

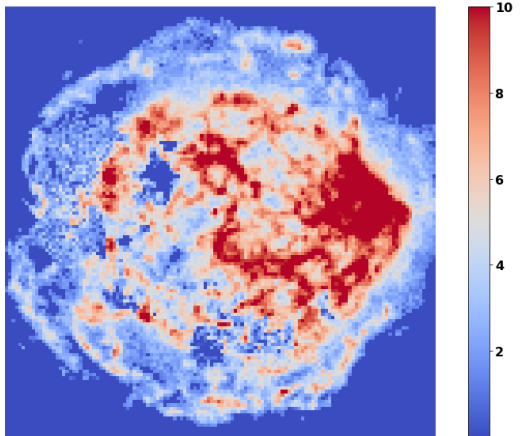

(f) Residual error

Figure 11: Sources estimated with HALS.

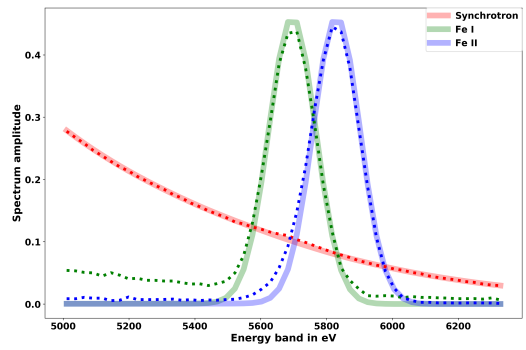

(a) GMCA

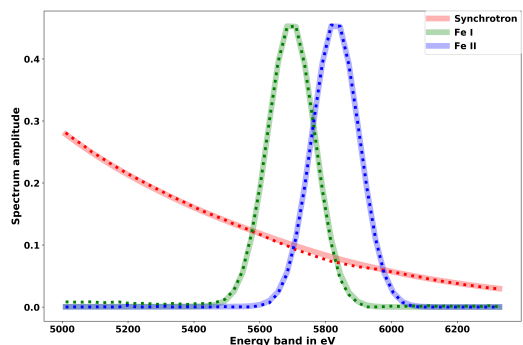

(b) pGMCA

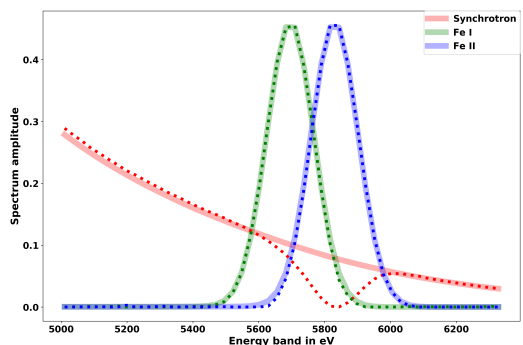

(c) HALS

Figure 12: Input (solid lines) and estimated spectra (dashed lines) with GMCA, pGMCA and HALS.

[15], such methods will be hampered by the Poisson nature of the measurements even for low number of counts. In that case, the ability to account for the exact statistics of the noise allows to mitigate this effect; this leads to a SAD that is one order of magnitude below than the other methods for a mean number of counts of 100 .

\section{Evolution of the relative intensity of the sources}

In this paragraph, we investigate the impact of the relative intensity of the sources on the separation process. More precisely, in the present astrophysical setting, the synchrotron emission can be regarded as a contamination with respect to the two redshifted iron sources. The accuracy of the estimated sources will therefore depend on the level of the synchrotron. In contrast to the standard additive Gaussian noise, this is particularly challenging when the observations are generated according to a Poisson distribution since the resulting stochasticity (or Poisson noise) will be dependent on the dominating source. This will hinder the separation process to a large extent. As a consequence, we evaluate the ability of the different BSS methods to accurately estimate the two iron components when the relative intensity of the synchrotron evolves.

Numerical experiments have been carried out with two different values for the number of observations: $m=12$ and $m=50$. In fact, for a fixed mean number of counts, estimating a fixed number of sources from a larger number of sources implies an increasing dimension reduction factor during the 


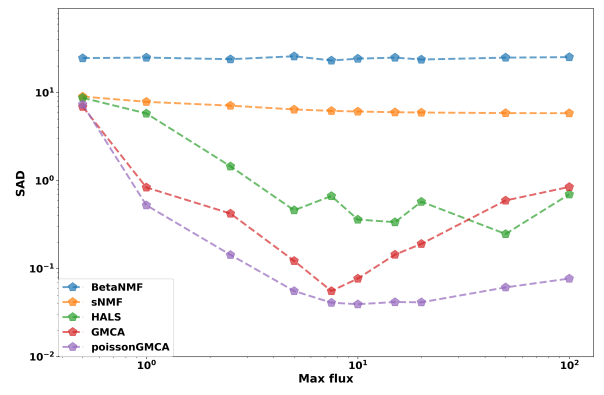

Figure 13: Spectral angular distance as a function of the mean flux.

separation process. In the Poisson statistics case, this entails that the noise that contaminates the sources will tend to "Gaussianize" as stated by the law of large numbers when the number of observations soars. This should be a more favorable scenario for standard BSS methods.

Case $m=12$ : The left panel of Fig. 14 features the evolution of the SAD when the parameter $\phi$ varies between 0.1 and 10 for a fixed number of observations $m=12$. In this experiment, the Poisson average evolves in the range [10,655]. First, the HALS method provide decent results with an average SAD of about $1^{\circ}$. Interestingly, the sNMF algorithm leads to rather reasonable values of the SAD when the level of synchrotron emission if low (i.e. $\rho<0.5$ ): in this regime, the two iron sources, which turn to be the sparsest ones, are dominant.

Both the GMCA and pGMCA algorithms lead to their best results for $\rho=0.3$ when the three sources share quite similar amplitudes. At high synchrotron level, both methods perform similarly with a SAD of $1^{\circ}$ when the synchroton emission is ten times larger $(\rho=10)$. In the range $[0.1,2]$, the pGMCA algorithm provides significantly cleaner separation results with a SAD that is more than one order than the other methods, which highlights the gain obtained by accounting for the exact Poisson nature of the measurements.

Case $m=50$ : The right panel of Fig. 14 shows the value of the SAD for $\phi \in[0.5$ and 10$]$. In this experiment, the mean number of counts evolves in the range [3, 334]. For a larger number of observations $m=50$, classical BSS methods perform slightly better than for $m=12$ while the average number of counts is two times lower. This is especially visible for large levels of the synchrotron emission. More interestingly, compared to GMCA, the pGMCA algorithm provides slightly worse results, especially for $\rho=2$. Indeed, as we pointed out previously, as the number of observations increase, standard BSS methods are less hampered by the Poisson statistics of the measurements. Still, the pGMCA yields separation results with a SAD gain up to one order of magnitude for $\rho=3.5$.

\section{ACKNOWLEDGEMENT}

This work is supported by the European Community through the grant LENA (ERC StG - contract no. 678282).

\section{Conclusion}

We investigated a new sparsity enforcing method to tackle blind source separation problems from measurements that follow Poisson statistics. Switching from the classical additive Gaussian noise to Poisson statistics entails key challenges: i) as a non-convex problem, the optimization strategy has a strong impact on the efficiency and robustness of the separation process especially when sparse regularization and Poisson likelihood lead to a non-smooth problem, ii) automatic parameter tuning becomes highly difficult in the case of Poisson statistics. For that purpose, the proposed pGMCA algorithm builds upon a BCD-like minimization scheme to optimize an approximate problem with a smooth data fidelity term. For that purpose, it is proposed to make use of Nesterov's smoothing technique to build a differentiable approximation of the Poisson loglikelihood. We further show that the proposed approach allows to define a simple and robust strategy to automatically tune the regularization parameters. Numerical experiments are carried out on both synthetic 1D spectroscopic data and realistic 2D simulations of astrophysical X-ray images. The results show a clear improvement of the separation quality with respect to existing methods in these different settings.

\section{REFERENCES}

[1] P. Comon and C. Jutten, Handbook of blind source separation, Academic Press, 2010.

[2] Cédric Févotte and Nicolas Dobigeon, "Nonlinear hyperspectral unmixing with robust nonnegative matrix factorization," IEEE Transactions on Image Processing, vol. 24, no. 12, pp. 4810-4819, 2015.

[3] Michael Zibulevsky and Barak A Pearlmutter, "Blind source separation by sparse decomposition in a signal dictionary," Neural computation, vol. 13 , no. 4, pp. 863-882, 2001

[4] Jérôme Bobin, Jean-Luc Starck, Jalal M Fadili, and Yassir Moudden, "Sparsity and morphological diversity in blind source separation," IEEE Transactions on Image Processing, vol. 16, no. 11, pp. 2662-2674, 2007.

[5] Carles Badenes, "X-ray studies of supernova remnants: A different view of supernova explosions," PNAS, vol. 107, no. 16, 2010.

[6] Minami Mihoko and Shinto Eguchi, "Robust blind source separation by beta divergence," Neural computation, vol. 14, no. 8, pp. 1859-1886, 2002.

[7] C. Févotte and J Idier, "Algorithms for nonnegative matrix factorization with the beta- divergence," Neural computation, vol. 23, no. 9, pp 2421-2456, 2011.

[8] J. Eggert and E Korner, "Sparse coding and nmf," in Proc. of Neural Networks, 2004

[9] Patrik O Hoyer, "Non-negative sparse coding," in Neural Networks for Signal Processing, 2002. Proceedings of the 2002 12th IEEE Workshop on. IEEE, 2002, pp. 557-565.

[10] J. Leroux, F Weninger, and J.R Hershey, "Sparse nmf - half-baked or well done?," Tech. Rep., Mitsubishi Electric Research Laboratories Technical Report, 2015. 


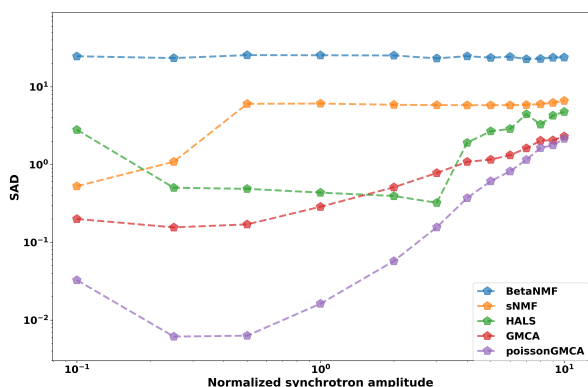

(a) Case $m=12$

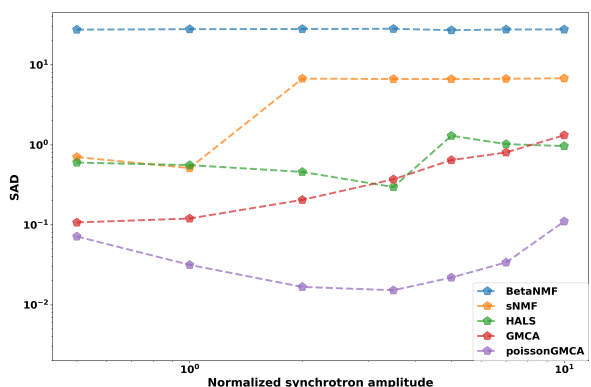

(b) Case $m=50$

Figure 14: Spectral angular distance as a function of the synchrotron level - left: $m=12$, right: case $m=50$.

[11] M. Zibulevski, "Blind source separation with relative Newton method," Proceedings of ICA, Independent Component Analysis, 2003, 2003, 897 902.

[12] A.M. Bronstein, M.M. Bronstein, M. Zibulevsky, and Y.Y. Zeevi, "Sparse ICA for blind separation of transmitted and reflected images," International Journal of Imaging Science and Technology, vol. 15/1, 2005, 84-91.

[13] Yuanqing Li, S.Amari, A.Cichocki, and Cuntai Guan, "Underdetermined blind source separation based on sparse representation," vol. 52, 2006, 3139-3152.

[14] J. Bobin, J.-L. Starck, J. Fadili, and Y. Moudden, "Sparsity and morphological diversity in blind source separation," Image Processing, IEEE Transactions on, vol. 16, no. 11, pp. 2662 -2674, nov. 2007.

[15] Jerome Bobin, Jeremy Rapin, Anthony Larue, and Jean-Luc Starck, "Sparsity and adaptivity for the blind separation of partially correlated sources.," IEEE Transanctions on Signal Processing, vol. 63, no. 5, pp. 1199-1213, 2015.

[16] J. Bobin, F. Sureau, J.-L. Starck, A. Rassat, and P. Paykari, "Joint planck and wmap cmb map reconstruction," Astronomy and Astrophysics, vol. 563, no. A105, 2014.

[17] Yangyang $\mathrm{Xu}$ and Wotao Yin, "A globally convergent algorithm for nonconvex optimization based on block coordinate update," arXiv preprint arXiv:1410.1386, 2014.

[18] Paul Tseng, "Convergence of a block coordinate descent method for nondifferentiable minimizations," Journal of Optimization Theory and Applications, vol. 3, no. 109, 2001.

[19] Yu Nesterov, "Smooth minimization of non-smooth functions," Mathe matical programming, vol. 103, no. 1, pp. 127-152, 2005.

[20] E. J. Candes, M. B. Wakin, and S. P. Boyd, "Enhancing sparsity by reweighted L1 minimization," vol. 14, no. 5, 2008, 877-905.

[21] Jeremy Rapin, Jerome Bobin, Anthony Larue, and Jean-Luc Starck, "NMF with Sparse Regularizations in Transformed Domains," SIAM Journal on Imaging Sciences (accepted), vol. 7, no. 4, pp. 2020-2047, 2014, SIAM J. Imaging Sci., 7(4), 2020-2047. (28 pages).

[22] Jérôme Bolte, Shoham Sabach, and Marc Teboulle, "Proximal alternating linearized minimization for nonconvex and nonsmooth problems," Mathematical Programming, vol. 146, no. 1-2, pp. 459-494, 2014.

[23] T. Rockafellar, Convex analysis, Princeton Landmarks in Mathematics and Physics. Princeton University Press, 1970.

[24] Neal Parikh, Stephen Boyd, et al., "Proximal algorithms," Foundations and Trends® in Optimization, vol. 1, no. 3, pp. 127-239, 2014.

[25] Patrick L Combettes and Jean-Christophe Pesquet, "Proximal splitting methods in signal processing," 2011.

[26] Hugo Raguet, Jalal Fadili, and Gabriel Peyré, "A generalized forwardbackward splitting," SIAM Journal on Imaging Sciences, vol. 6, no. 3, pp. 1199-1226, 2013.

[27] J.-L.Starck, J.Fadili, and F.Murtagh, "The undecimated wavelet decomposition and its reconstruction," IEEE Transactions on Image Processing, vol. 16, pp. 297-309, 2007.

[28] J.-L. Starck, E.J. Candès, and D.L. Donoho, "The curvelet transform for image denoising," IEEE Transactions on Image Processing, vol. 11, no. 6, pp. 670-684, 2002.

[29] Amir Beck and Marc Teboulle, "A fast iterative shrinkage-thresholding algorithm for linear inverse problems," SIAM journal on imaging sciences, vol. 2, no. 1, pp. 183-202, 2009.
[30] C. Kervazo, J. Bobin, and C. Chenot, "Heuristics for efficient sparse blind source separation," in 8th International Conference on New Computational Methods for Inverse Problems 25 May 2018, Cachan, France, 2018, vol. 1131.

[31] Jean-Luc Starck, DL Donoho, and M Elad, "Redundant multiscale transforms and their application for morphological component separation," 2004.

[32] N. Gillis and F. Glineur, "Accelerated Multiplicative Updates and Hierarchical ALS Algorithms for Nonnegative Matrix Factorization," Neural Computation, vol. 24, no. 4, pp. 1085-1105, 2012.

[33] Emmanuel Vincent, Remi Gribonval, and Cedric Fevotte, "Performance measurement in blind audio source separation," IEEE Transactions on Audio, Speech \& Language Processing, vol. 14, no. 4, pp. 1462-1469, 2006. 